\title{
Caenorhabditis elegans as a Promising Alternative Model for Environmental Chemical Mixture Effect Assessment - A Comparative Study
}

Paul Wittkowski, ${ }^{\dagger}$ Philip Marx-Stoelting, ${ }^{\dagger}$ Norman Violet, ${ }^{\dagger}$ Verena Fetz, ${ }^{\dagger}$ Franziska Schwarz, ${ }^{\dagger}$ Michael Oelgeschläger, ${ }^{\dagger}$ Gilbert Schönfelder, ${ }^{+, \ddagger}$ Silvia Vogl ${ }^{*, \dagger}$

${ }^{\dagger}$ German Federal Institute for Risk Assessment, German Centre for the Protection of Laboratory Animals (Bf 3 R), 10589 Berlin, Germany

${ }^{\ddagger}$ Charité - Universitätsmedizin Berlin, corporate member of Freie Universität Berlin, HumboldtUniversität zu Berlin, and Berlin Institute of Health, 10117 Berlin, Germany

* corresponding author

Address: Max-Dohrn-Str. 8-10, 10589 Berlin, Germany

Phone: +49-30-18412-0

e-mail: silvia.vogl@bfr.bund.de

Number of Pages: 19

Number of Figures: 6

Number of Tables: 12 


\section{Detailed Description of Developmental and Reproduction Test}

To synchronize worms, a three day old N2 liquid culture (4000 worms in $10 \mathrm{~mL}$ liquid medium, $10^{9} \mathrm{cfu} / \mathrm{mL}$ OP50, $20^{\circ} \mathrm{C}$ ) was treated with bleach solution following standard protocols for egg preparation. In brief, the culture was washed twice with M9-buffer $(5 \mathrm{~g}$ $\mathrm{NaCl}, 3 \mathrm{~g} \mathrm{KH}_{2} \mathrm{PO}_{4}, 11.3 \mathrm{~g} \mathrm{Na}_{2} \mathrm{HPO}_{4}, \mathrm{H}_{2} \mathrm{O}$ to $1 \mathrm{~L}$ autoclaved, after cooling, add $1 \mathrm{~mL}$ sterile $1 \mathrm{M} \mathrm{MgSO}_{4}$ ), centrifuged (2 $\mathrm{min}, 1300 \mathrm{~g}$ ) and the supernatant was removed, leaving a volume of $2 \mathrm{~mL}$. After adding $250 \mu \mathrm{L} 10 \mathrm{~N} \mathrm{NaOH}$ and $420 \mu \mathrm{L} 12 \% \mathrm{NaOCl}$, the tubes were shaken vigorously for approximately $8 \mathrm{~min}$ until all adult worms were dissolved and only eggs remained. The eggs were washed three times with M9 buffer and then left to hatch in $3 \mathrm{~mL}$ liquid medium (S-complete: $5.85 \mathrm{~g} / \mathrm{L} \mathrm{NaCl}, 1 \mathrm{~g} / \mathrm{L} \mathrm{K}{ }_{2} \mathrm{PO}_{4}, 6 \mathrm{~g} / \mathrm{L} \mathrm{KH}_{2} \mathrm{PO}_{4}, 10 \mathrm{mM}$ potassium citrate, $1 \% \mathrm{v} / \mathrm{v}$ trace metals solution $\left(\mathrm{Na}_{2} \mathrm{EDTA} 1.68 \mathrm{~g} / \mathrm{L}, \mathrm{Fe}(\mathrm{II}) \mathrm{SO}_{4} 0.377 \mathrm{~g} / \mathrm{L}, \mathrm{Mn}(\mathrm{II}) \mathrm{Cl}_{2}\right.$ $\left.0.127 \mathrm{~g} / \mathrm{L}, \mathrm{ZnSO}_{4} 0.163 \mathrm{~g} / \mathrm{L}, \mathrm{Cu}(\mathrm{II}) \mathrm{SO}_{4} 0.016 \mathrm{~g} / \mathrm{L}\right), 3 \mathrm{mM} \mathrm{CaCl}, 3 \mathrm{mM} \mathrm{MgSO} 4,5 \mathrm{mg} / \mathrm{L}$ cholesterol in $\mathrm{H}_{2} \mathrm{O}$ ) without feeding bacteria at $20{ }^{\circ} \mathrm{C}$. After at least $12 \mathrm{~h}$ hatching time, synchronized L1 larvae were transferred individually (one L1 per well) into $30 \mu \mathrm{L}$ S-complete in 96 well microtiter plates (CellCarrier 96, PerkinElmer Inc., MA, USA) under a stereomicroscope (Stemi 608, Carl Zeiss Microscopy) using a manual pipette set to $1 \mu \mathrm{L}$ (Eppendorf AG, HH, DE). Edge wells were filled with $150 \mu \mathrm{L} \mathrm{ddH}_{2} \mathrm{O}$. Finally, $120 \mu \mathrm{L}$ liquid medium with OP50 (final concentration $10^{9} \mathrm{cfu} / \mathrm{mL}$ ) and test substances (final concentration $0.2 \%$ DMSO) was added to each inner well. 60 replicates were used for solvent control and 30 per treatment. Treated and untreated worms were incubated in 96 well plates for $96 \mathrm{~h}$ at $20^{\circ} \mathrm{C}$ in the dark. Subsequently, $15 \mu \mathrm{L}$ rose bengal (300 mg/L) were added per well and plates were heated to $80^{\circ} \mathrm{C}$ for 20 min using a heating oven (Thermo Fisher Scientific). Stained food bacteria was removed by repetitively adding $100 \mu \mathrm{L}$ ddH2O per well and removing $100 \mu \mathrm{L}$ supernatant after $5 \mathrm{~min}$ of sedimentation at room temperature without aspirating worms and offspring using a multi-channel pipette. Microscopical inspection of the collected aspirated supernatant confirmed that almost no offspring were removed during the washing steps. Imaging was performed automatically by Opera Phenix High Content Screening System (PerkinElmer Inc., MA, USA). To visualize whole wells, a 5x air objective 
(NA 0.16, Filter: Cy3; emission: $570-630 \mathrm{~nm}$; excitation: $561 \mathrm{~nm}$, illumination time: $100 \mathrm{~ms}$ at $100 \%$ intensity) was used leading to 9 sub-images per well. The sub-images were merged without overlap for whole well images. Merged images were imported into the open-source software CellProfiler v.2.2.0 (Carpenter Lab MIT, MA, USA) and stained worms were measured (worm area) and their offspring (eggs and larvae) was counted automatically out of binarized images (Otsu-method).

\section{Test Substances and Solvent Control}

Table S1: Test substances

\begin{tabular}{ccccc} 
& CAS \# & $\begin{array}{c}\text { Batch \# } \\
\text { or Lot \# }\end{array}$ & $\begin{array}{c}\text { Purity } \\
\%\end{array}$ & Purchased from \\
\hline $\begin{array}{c}\text { epoxiconazole } \\
\text { prochloraz }\end{array}$ & $133855-98-8$ & $5154 \mathrm{X}$ & 97.0 & BASF (Ludwigshafen, Germany) \\
cyproconazole & $67747-09-5$ & COD-000718 & 98.0 & BASF (Ludwigshafen, Germany) \\
propiconazole & $60207-90-1$ & CGA64250B & 96.10 & Syngenta (Basel, Switzerland) \\
tebuconazole & $107534-96-3$ & NK21BX0392 & 96.20 & Bayer CropScience (Monheim, \\
& & & & Germany) \\
\hline
\end{tabular}

Table S2: Azole fungicide dilution series of single chemicals and binary combinations in a fixed ratio design used in the $96 \mathrm{~h}$ assay

[ $\mu \mathrm{M}]$ conc. 1 conc. 2 conc. 3 conc. 4 conc. 5 conc. 6 conc. 7 conc. 8

\begin{tabular}{ccccccccc}
\hline epoxiconazole & 0.09 & 0.19 & 0.38 & 0.76 & 1.52 & 3.03 & 6.07 & 12.13 \\
prochloraz & 0.08 & 0.17 & 0.33 & 0.66 & 1.33 & 2.65 & 5.31 & 10.62 \\
cyproconazole & 2.68 & 5.36 & 10.71 & 21.42 & 42.84 & 85.68 & 171.36 & 342.72 \\
propiconazole & 2.28 & 4.57 & 9.13 & 18.26 & 36.53 & 73.05 & 146.10 & 292.21 \\
tebuconazole & 0.76 & 1.52 & 3.05 & 6.09 & 12.18 & 24.36 & 48.73 & 97.46 \\
\hline epoxiconazole & 0.09 & 0.19 & 0.38 & 0.76 & 1.52 & 3.03 & 6.07 & 12.13 \\
+ & + & + & + & + & + & + & + & + \\
prochloraz & 0.08 & 0.17 & 0.33 & 0.66 & 1.33 & 2.65 & 5.31 & 10.62 \\
epoxiconazole & 0.09 & 0.19 & 0.38 & 0.76 & 1.52 & 3.03 & 6.07 & 12.13 \\
+ & + & + & + & + & + & + & + & + \\
cyproconazole & 2.68 & 5.36 & 10.71 & 21.42 & 42.84 & 85.68 & 171.36 & 342.72 \\
\hline
\end{tabular}




\begin{tabular}{|c|c|c|c|c|c|c|c|c|}
\hline epoxiconazole & 0.09 & 0.19 & 0.38 & 0.76 & 1.52 & 3.03 & 6.07 & 12.13 \\
\hline+ & + & + & + & + & + & + & + & + \\
\hline propiconazole & 2.28 & 4.57 & 9.13 & 18.26 & 36.53 & 73.05 & 146.10 & 292.21 \\
\hline epoxiconazole & 0.09 & 0.19 & 0.38 & 0.76 & 1.52 & 3.03 & 6.07 & 12.13 \\
\hline+ & + & + & + & + & + & + & + & + \\
\hline tebuconazole & 0.76 & 1.52 & 3.05 & 6.09 & 12.18 & 24.36 & 48.73 & 97.46 \\
\hline prochloraz & 0.08 & 0.17 & 0.33 & 0.66 & 1.33 & 2.65 & 5.31 & 10.62 \\
\hline+ & + & + & + & + & + & + & + & + \\
\hline cyproconazole & 2.68 & 5.36 & 10.71 & 21.42 & 42.84 & 85.68 & 171.36 & 342.72 \\
\hline prochloraz & 0.08 & 0.17 & 0.33 & 0.66 & 1.33 & 2.65 & 5.31 & 10.62 \\
\hline+ & + & + & + & + & + & + & + & + \\
\hline propiconazole & 2.28 & 4.57 & 9.13 & 18.26 & 36.53 & 73.05 & 146.10 & 292.21 \\
\hline prochloraz & 0.08 & 0.17 & 0.33 & 0.66 & 1.33 & 2.65 & 5.31 & 10.62 \\
\hline+ & + & + & + & + & + & + & + & + \\
\hline tebuconazole & 0.76 & 1.52 & 3.05 & 6.09 & 12.18 & 24.36 & 48.73 & 97.46 \\
\hline cyproconazole & 2.68 & 5.36 & 10.71 & 21.42 & 42.84 & 85.68 & 171.36 & 342.72 \\
\hline+ & + & + & + & + & + & + & + & + \\
\hline propiconazole & 2.28 & 4.57 & 9.13 & 18.26 & 36.53 & 73.05 & 146.10 & 292.21 \\
\hline cyproconazole & 2.68 & 5.36 & 10.71 & 21.42 & 42.84 & 85.68 & 171.36 & 342.72 \\
\hline+ & + & + & + & + & + & + & + & + \\
\hline tebuconazole & 0.76 & 1.52 & 3.05 & 6.09 & 12.18 & 24.36 & 48.73 & 97.46 \\
\hline propiconazole & 2.28 & 4.57 & 9.13 & 18.26 & 36.53 & 73.05 & 146.10 & 292.21 \\
\hline+ & + & + & + & + & + & + & + & + \\
\hline tebuconazole & 0.76 & 1.52 & 3.05 & 6.09 & 12.18 & 24.36 & 48.73 & 97.46 \\
\hline
\end{tabular}




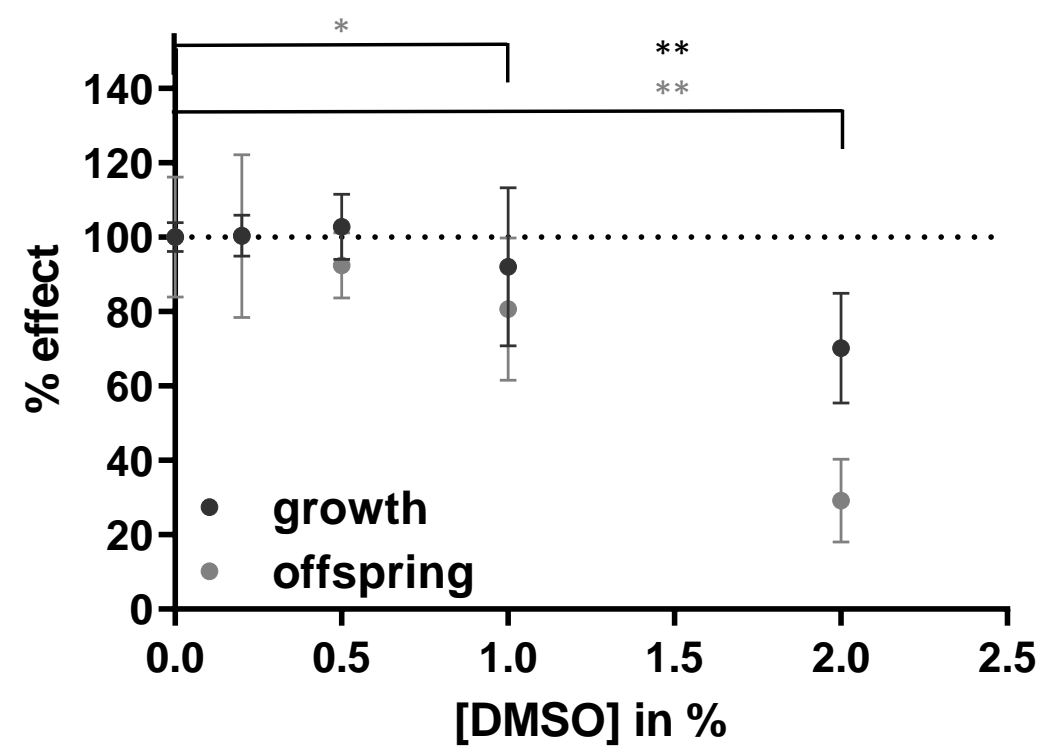

Figure S1: Growth inhibition and offspring reduction of solvent DMSO. Points depict mean \pm SD ( $n \leq 12)$. Statistical analysis against control without DMSO via one-way-ANOVA with Dunnett correction $(p \leq 0.05)$. ${ }^{*} p \leq 0.05 ;{ }^{* *} p \leq 0.0001$

Statistically significant effects on reproduction were found at $1 \%$ DMSO ( $p=0.0265$, oneway-ANOVA with post-hoc Dunnett test) and on growth at $2 \%$ DMSO $(p<0.0001)$. No significant effects on growth $(p=0.999)$ and reproduction $(p>0.999)$ were found at the chosen test concentration of $0.2 \%$ DMSO.

Detailed Description of the Methods for Analytical Quantification of Azole Fungicides

\section{Sample Collection and Preparation}

A three day old N2 liquid culture (4000 worms in $10 \mathrm{~mL}$ liquid medium, $10^{9} \mathrm{cfu} / \mathrm{mL}$ OP50) was synchronized by egg preparation following standard procedures with bleach solution (for more detailed description, see S1). After at least $12 \mathrm{~h}$ hatching time, synchronized L1 larvae were counted in five drops of $5 \mu \mathrm{L}$ hatching culture. The respective volume containing $400 \mathrm{~L} 1$ larvae was transferred to culture flasks and the worms treated with $1 \mu \mathrm{g} / \mathrm{mL}$ epoxiconazole, prochloraz, cyproconazole, propiconazole and tebuconazole alone and in all ten binary 
combinations in a final volume of $10 \mathrm{~mL}$ liquid medium with $10^{9} \mathrm{cfu} / \mathrm{mL}$ OP50 for $96 \mathrm{~h}$ at $20^{\circ} \mathrm{C}$ in the dark. The supernatant was collected and diluted $1: 100$ in $\mathrm{dd}_{2} \mathrm{O}$. Extraction of supernatants was performed in three steps with dichloromethane and subsequently the solvent was evaporated to dryness under a nitrogen stream. Sedimented worms were washed three times in $10 \mathrm{~mL}$ M9 buffer and supernatant was removed. Lysis of the pellets was performed in a three-step process by adding $500 \mu \mathrm{L}$ acetone and a mixture of steel and ceramic beads (1.4 - $1.6 \mathrm{~mm}$ and $3.5 \mathrm{~mm}$, Analytik Jena AG, Jena, Germany) in a SpeedMill Plus (Analytik Jena AG) for 2 min. The solvent of the combined extracts was removed under a nitrogen stream and all samples were diluted in acetonitrile for analytical quantification via LC-MS/MS.

\section{Chemical Analysis}

Analyses were performed using HPLC system LC-20A Prominence (Shimadzu Corporation, Jena, Germany) (component list: Table S3). Separation was achieved with a Kinetex®

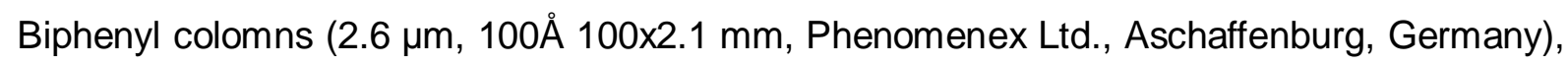
with a flow rate of $0.4 \mathrm{~mL} / \mathrm{min}$ and an injection volume of $5 \mu \mathrm{L}$ (gradient program: Table S4). Autosampler was cooled to $15^{\circ} \mathrm{C}$ and oven temperature was $40^{\circ} \mathrm{C}$. For mass-spectrometric analysis MDS Sciex 4000 QTRAP (TIS/ESI) (Applied Biosystems Thermo Fisher Scientific, MA, USA) was used (MRMs: Table S5).

Table S3: HPLC system components

\begin{tabular}{cl}
\hline device & \multicolumn{1}{c}{ manufacturer } \\
\hline autosampler SIL-20ACXR & Shimadzu Corporation (Kyoto, Japan) \\
column oven CTO-20ACXR & Shimadzu Corporation (Kyoto, Japan) \\
controller CBM-20A & Shimadzu Corporation (Kyoto, Japan) \\
mass spectrometer MDS Sciex 4000 QTRAP (TIS/ESI) & Thermo Fisher Scientific (Waltham, US) \\
pump LC-20ADXR & Shimadzu Corporation (Kyoto, Japan) \\
vaccum degasser DGU-20A5 & Shimadzu Corporation (Kyoto, Japan) \\
\hline
\end{tabular}


Table S4: HPLC gradient program

Solution A: $90: 10 \mathrm{MeOH} / \mathrm{H}_{2} \mathrm{O}(\mathrm{v} / \mathrm{v}), 5 \mathrm{mM}$ ammonium formate, $0.1 \%$ acetic acid

Solution $\mathrm{B}: \mathrm{H}_{2} \mathrm{O}, 5 \mathrm{mM}$ ammonium formate, $0.1 \%$ acetic acid

\begin{tabular}{cccc}
\hline & time [min] & solution A [\%] & solution B [\%] \\
\hline start & 0.01 & & \\
& 0.02 & 10 & 90 \\
injection & 0.03 & & \\
& 0.5 & 100 & 0 \\
& 5 & 100 & 0 \\
& 6.1 & 10 & 90 \\
end & 8 & 10 & 90 \\
& 8.1 & & \\
\hline
\end{tabular}

Table S5: mass transitions of azole fungicides and internal standards

DP (declustering potential), CE (collision energy), CXP (cell exit potential)

\begin{tabular}{|c|c|c|c|c|}
\hline analyte & mass transition & $\mathrm{DP}[\mathrm{V}]$ & CE [V] & CXP [V] \\
\hline Epoxiconazol 1 & $330.045 \rightarrow 75.100$ & 71 & 111 & 0 \\
\hline Epoxiconazol 2 & $330.045 \rightarrow 101.200$ & 71 & 71 & 4 \\
\hline Epoxiconazol-d $d_{4}$ & $334.078 \rightarrow 77.000$ & 66 & 105 & 10 \\
\hline Prochloraz 1 & $376.070 \rightarrow 307.900$ & 56 & 19 & 8 \\
\hline Prochloraz 2 & $376.070 \rightarrow 70.000$ & 56 & 43 & 10 \\
\hline Prochloraz 3 & $376.070 \rightarrow 43.000$ & 56 & 67 & 6 \\
\hline Prochloraz-d & $383.908 \rightarrow 316.100$ & 51 & 19 & 6 \\
\hline Cyproconazol 1 & $292.090 \rightarrow 70.000$ & 76 & 45 & 10 \\
\hline Cyproconazol 2 & $292.090 \rightarrow 125.000$ & 76 & 45 & 8 \\
\hline Cyproconazol 3 & $292.090 \rightarrow 89.000$ & 76 & 89 & 14 \\
\hline Triadimenol & $296.100 \rightarrow 69.900$ & 46 & 31 & 12 \\
\hline Propiconazol 1 & $342.010 \rightarrow 158.800$ & 91 & 39 & 12 \\
\hline Propiconazol 2 & $342.010 \rightarrow 69.000$ & 91 & 37 & 10 \\
\hline Propiconazol 3 & $342.010 \rightarrow 41.000$ & 91 & 65 & 6 \\
\hline Propiconazol-d $d_{5}$ & $348.022 \rightarrow 159.000$ & 81 & 51 & 10 \\
\hline Tebuconazol 1 & $308.060 \rightarrow 69.900$ & 81 & 49 & 10 \\
\hline Tebuconazol 2 & $308.060 \rightarrow 125.000$ & 81 & 53 & 8 \\
\hline Tebuconazol 3 & $308.060 \rightarrow 42.900$ & 81 & 115 & 6 \\
\hline Tebuconazol- $\mathrm{d}_{6}$ & $314.160 \rightarrow 72.000$ & 81 & 51 & 12 \\
\hline
\end{tabular}




\section{Quantification and Quality Control}

Prior to extraction, internal standard mix was added to all samples to reach a final concentration of $0.2 \mathrm{ng} / \mu \mathrm{L}$ (triadimenol, propiconazole- $\mathrm{d}_{5}$ ) or $0.02 \mathrm{ng} / \mu \mathrm{L}$ (epoxiconazole- $\mathrm{d}_{4}$, prochloraz- $d_{7}$, tebuconazole- $d_{6}$ ) for each analyte. For quantification, two calibration curves were used for each sample type in the concentration range $25 \mathrm{ng} / \mathrm{mL}-1.5 \mu \mathrm{g} / \mathrm{mL}$ (supernatant) and $3.125 \mathrm{ng}-37.5 \mathrm{ng}$ (pellets). All signals were quantified with MultiQuant 2.1.1. (AB Sciex Pte. Ltd., Woodlands, SGP). Linear regression and a weighting factor $1 / x$ offered best fits to the calibration curves (Table S4 and S5). Measured pellet residues were used to calculate the internal azole concentrations in the worms (in $\mu \mathrm{M}$ ), assuming a volume of $2 \mu \mathrm{L}$ per 400 worms. $^{1}$

Table S6: Analytical quantification of azole fungicides: limits of detection (LODs) and limits of quantification (LOQs) in the supernatant (medium and food bacteria) [ng/ml] and in the worm pellets [pg]

Supernatant Worm pellet

LOD LOQ LOD LOQ

$\begin{array}{ccccc}\text { epoxiconazole } & 0.5 & 2.5 & <62.5 & <62.5 \\ \text { prochloraz } & 0.25 & 2.5 & <62.5 & <62.5 \\ \text { cyproconazole } & 2.5 & 10 & 62.5 & 312.5 \\ \text { propiconazole } & 1.0 & 2.5 & <62.5 & <62.5 \\ \text { tebuconazole } & 2.5 & 10 & <62.5 & <62.5\end{array}$


Table S7: Analytical quantification of azole fungicides: reproducibility of the supernatant calibration standards (VC = variation coefficient; acc = accuracy)

\begin{tabular}{|c|c|c|c|c|c|c|c|c|c|}
\hline & \multirow{3}{*}{$\begin{array}{c}\text { Expected } \\
\text { concentration } \\
{[\mathrm{ng} / \mathrm{mL}]}\end{array}$} & \multicolumn{4}{|c|}{ Intraday $(n=3)$} & \multicolumn{4}{|c|}{ Interday $(n=4)$} \\
\hline & & Mean & SD & VC & acc & Mean & $\overline{S D}$ & VC & acc \\
\hline & & & & $\%$ & $\%$ & & & $\%$ & $\%$ \\
\hline \multirow[t]{9}{*}{ epoxiconazole } & 25 & 22.7 & 3.4 & 14.9 & 90.9 & 27.3 & 2.9 & 10.8 & 109.1 \\
\hline & 50 & 52.7 & 4.6 & 8.8 & 105.4 & 56.7 & 3.7 & 6.5 & 113.4 \\
\hline & 125 & 126.3 & 6.8 & 5.4 & 101.0 & 133.0 & 1.8 & 1.4 & 106.4 \\
\hline & 250 & 253.1 & 15.0 & 5.9 & 101.3 & 266.5 & 2.6 & 1.0 & 106.6 \\
\hline & 500 & 492.6 & 0.2 & 0.0 & 98.5 & 503.1 & 10.4 & 2.1 & 100.6 \\
\hline & 750 & 739.9 & 20.9 & 2.8 & 98.7 & 729.0 & 5.6 & 0.8 & 97.2 \\
\hline & 1000 & 1006.8 & 16.1 & 1.6 & 100.7 & 985.2 & 17.1 & 1.7 & 98.5 \\
\hline & 1250 & 1270.0 & 97.7 & 7.7 & 101.6 & 1390.6 & 43.0 & 3.1 & 111.2 \\
\hline & 1500 & 1486.8 & 50.5 & 3.4 & 99.1 & 1511.1 & 13.4 & 0.9 & 100.7 \\
\hline \multirow[t]{9}{*}{ prochloraz } & 25 & 22.5 & 4.5 & 20.0 & 90.0 & 24.4 & 0.0 & 0.1 & 97.5 \\
\hline & 50 & 53.1 & 5.1 & 9.6 & 106.2 & 56.5 & 3.0 & 5.3 & 112.9 \\
\hline & 125 & 123.1 & 7.8 & 6.3 & 98.5 & 131.5 & 4.0 & 3.0 & 105.2 \\
\hline & 250 & 255.0 & 23.9 & 9.4 & 102.0 & 265.4 & 5.4 & 2.0 & 106.2 \\
\hline & 500 & 519.7 & 20.8 & 4.0 & 103.9 & 506.0 & 10.9 & 2.1 & 101.2 \\
\hline & 750 & 729.2 & 12.1 & 1.7 & 97.2 & 737.5 & 24.5 & 3.3 & 98.3 \\
\hline & 1000 & 964.5 & 19.1 & 2.0 & 96.4 & 941.7 & 16.1 & 1.7 & 94.2 \\
\hline & 1250 & 1269.7 & 103.6 & 8.2 & 101.6 & 1459.2 & 8.8 & 0.6 & 116.7 \\
\hline & 1500 & 1518.2 & 68.5 & 4.5 & 101.2 & 1453.2 & 56.3 & 3.9 & 96.9 \\
\hline \multirow[t]{9}{*}{ cyproconazole } & 25 & 18.8 & 1.7 & 8.9 & 75.4 & 18.1 & 0.4 & 2.5 & 72.3 \\
\hline & 50 & 50.1 & 3.2 & 6.4 & 100.2 & 53.5 & 1.8 & 3.3 & 106.9 \\
\hline & 125 & 148.4 & 13.1 & 8.8 & 118.7 & 138.6 & 1.4 & 1.0 & 110.8 \\
\hline & 250 & 259.0 & 22.6 & 8.7 & 103.6 & 279.0 & 5.8 & 2.1 & 111.6 \\
\hline & 500 & 489.2 & 15.2 & 3.1 & 97.8 & 500.7 & 9.2 & 1.8 & 100.1 \\
\hline & 750 & 724.7 & 52.9 & 7.3 & 96.6 & 692.7 & 17.7 & 2.6 & 92.4 \\
\hline & 1000 & 949.6 & 78.9 & 8.3 & 95.0 & 997.4 & 30.9 & 3.1 & 99.7 \\
\hline & 1250 & 1305.5 & 122.5 & 9.4 & 104.4 & 1452.2 & 27.8 & 1.9 & 116.2 \\
\hline & 1500 & 1508.4 & 33.9 & 2.2 & 100.6 & 1469.4 & 27.3 & 1.9 & 98.0 \\
\hline \multirow[t]{3}{*}{ propiconazole } & 25 & 22.5 & 4.2 & 18.5 & 90.1 & 25.9 & 0.2 & 0.8 & 103.6 \\
\hline & 50 & 53.0 & 4.7 & 8.8 & 105.9 & 55.3 & 3.2 & 5.8 & 110.6 \\
\hline & 125 & 124.8 & 9.9 & 7.9 & 99.9 & 132.9 & 4.6 & 3.5 & 106.3 \\
\hline
\end{tabular}




\begin{tabular}{cccccccccc}
\hline & 250 & 254.2 & 17.3 & 6.8 & 101.7 & 263.2 & 5.2 & 2.0 & 105.3 \\
& 500 & 501.7 & 6.0 & 1.2 & 100.3 & 501.8 & 15.1 & 3.0 & 100.4 \\
& 750 & 747.9 & 22.1 & 3.0 & 99.7 & 729.1 & 11.9 & 1.6 & 97.2 \\
& 1000 & 981.7 & 18.2 & 1.8 & 98.2 & 987.8 & 24.1 & 2.4 & 98.8 \\
& 1250 & 1252.2 & 122.5 & 9.8 & 100.2 & 1394.1 & 22.0 & 1.6 & 111.5 \\
& 1500 & 1514.5 & 25.5 & 1.7 & 101.0 & 1509.5 & 46.8 & 3.1 & 100.6 \\
\hline \multirow{3}{*}{ tebuconazole } & & & & & & & & & \\
& 25 & 23.9 & 1.6 & 6.6 & 95.5 & 27.8 & 0.7 & 2.4 & 111.2 \\
& 50 & 52.3 & 3.4 & 6.5 & 104.5 & 56.7 & 0.7 & 1.2 & 113.5 \\
& 125 & 121.9 & 5.5 & 4.5 & 97.6 & 133.9 & 2.5 & 1.9 & 107.2 \\
& 250 & 253.0 & 11.8 & 4.7 & 101.2 & 263.1 & 3.1 & 1.2 & 105.2 \\
& 500 & 495.0 & 15.5 & 3.1 & 99.0 & 500.8 & 5.9 & 1.2 & 100.1 \\
& 750 & 768.6 & 14.6 & 1.9 & 102.5 & 754.0 & 7.0 & 0.9 & 100.5 \\
& 1000 & 980.8 & 8.1 & 0.8 & 98.1 & 968.3 & 26.5 & 2.7 & 96.8 \\
& 1250 & 1244.2 & 100.2 & 8.1 & 99.5 & 1357.8 & 40.9 & 3.0 & 108.6 \\
& 1500 & 1512.2 & 30.8 & 2.0 & 100.8 & 1468.8 & 32.4 & 2.2 & 97.9 \\
\hline
\end{tabular}

Table S8: Analytical quantification of azole fungicides: reproducibility of the pellet calibration standards $(\mathrm{VC}=$ variation coefficient $;$ acc $=$ accuracy $)$. Substance amount in 400 worms .

Expected concentration [ng]

3.125

6.25

12.5

18.75

25

31.25

37.5
Intraday $(\mathrm{n}=3)$

Interday ( $=4)$

\begin{tabular}{cccccccc}
\hline Mean & SD & $\begin{array}{c}\text { VC } \\
\%\end{array}$ & $\begin{array}{c}\text { acc } \\
\%\end{array}$ & Mean & SD & $\begin{array}{c}\text { VC } \\
\%\end{array}$ & $\begin{array}{c}\text { acc } \\
\%\end{array}$ \\
\hline & & & & & & & \\
2.7 & 0.2 & 8.1 & 87.5 & 2.8 & 0.2 & 7.2 & 87.8 \\
6.3 & 0.5 & 7.8 & 101.4 & 6.5 & 0.5 & 7.4 & 103.7 \\
12.9 & 0.9 & 7.2 & 102.8 & 13.5 & 0.6 & 4.7 & 108.4 \\
18.8 & 0.6 & 3.1 & 100.5 & 19.3 & 1.0 & 4.9 & 103.1 \\
24.4 & 1.1 & 4.7 & 97.6 & 25.0 & 0.7 & 2.9 & 99.8 \\
32.1 & 1.7 & 5.3 & 102.8 & 33.1 & 1.6 & 4.9 & 106.0 \\
37.0 & 0.7 & 1.8 & 98.7 & 37.6 & 1.2 & 3.1 & 100.2
\end{tabular}




\begin{tabular}{|c|c|c|c|c|c|c|c|c|c|}
\hline \multirow[t]{7}{*}{ prochloraz } & 3.125 & 2.6 & 0.1 & 3.1 & 84.6 & 2.6 & 0.0 & 1.3 & 83.3 \\
\hline & 6.25 & 6.4 & 0.8 & 11.7 & 103.0 & 6.6 & 0.6 & 8.7 & 105.4 \\
\hline & 12.5 & 12.8 & 0.6 & 5.1 & 102.4 & 12.9 & 0.7 & 5.5 & 103.6 \\
\hline & 18.75 & 18.8 & 0.8 & 4.1 & 100.3 & 19.0 & 0.8 & 4.4 & 101.2 \\
\hline & 25 & 24.9 & 1.4 & 5.8 & 99.4 & 25.2 & 0.5 & 2.1 & 100.8 \\
\hline & 31.25 & 31.6 & 0.5 & 1.5 & 101.1 & 31.9 & 0.8 & 2.4 & 102.2 \\
\hline & 37.5 & 37.2 & 0.4 & 1.2 & 99.2 & 38.7 & 1.4 & 3.5 & 103.2 \\
\hline \multirow[t]{7}{*}{ cyproconazole } & 3.125 & 2.8 & 0.1 & 1.9 & 89.5 & 2.8 & 0.1 & 2.0 & 89.8 \\
\hline & 6.25 & 6.6 & 0.8 & 12.0 & 105.0 & 6.6 & 0.5 & 8.2 & 105.8 \\
\hline & 12.5 & 12.5 & 1.3 & 10.7 & 99.8 & 13.1 & 0.5 & 4.0 & 105.1 \\
\hline & 18.75 & 19.1 & 0.9 & 4.6 & 101.9 & 19.0 & 0.5 & 2.6 & 101.6 \\
\hline & 25 & 24.6 & 1.3 & 5.2 & 98.2 & 24.4 & 1.6 & 6.5 & 97.6 \\
\hline & 31.25 & 31.2 & 0.7 & 2.1 & 99.9 & 31.3 & 0.1 & 0.4 & 100.0 \\
\hline & 37.5 & 37.6 & 0.8 & 2.2 & 100.3 & 37.5 & 1.8 & 4.8 & 100.0 \\
\hline \multirow[t]{7}{*}{ propiconazole } & 3.125 & 2.7 & 0.1 & 2.8 & 86.9 & 2.8 & 0.0 & 0.4 & 88.5 \\
\hline & 6.25 & 6.5 & 0.6 & 9.1 & 104.7 & 6.6 & 0.6 & 9.4 & 106.3 \\
\hline & 12.5 & 12.8 & 0.2 & 1.8 & 102.0 & 12.8 & 0.2 & 1.3 & 102.5 \\
\hline & 18.75 & 19.0 & 0.7 & 3.6 & 101.3 & 19.3 & 0.5 & 2.3 & 102.9 \\
\hline & 25 & 24.3 & 1.2 & 5.0 & 97.1 & 25.1 & 1.6 & 6.4 & 100.6 \\
\hline & 31.25 & 31.5 & 0.9 & 2.9 & 100.7 & 31.6 & 0.7 & 2.2 & 101.3 \\
\hline & 37.5 & 37.6 & 1.0 & 2.7 & 100.2 & 38.8 & 0.5 & 1.3 & 103.5 \\
\hline \multirow[t]{7}{*}{ tebuconazole } & 3.125 & 2.8 & 0.0 & 1.4 & 89.2 & 2.6 & 0.3 & 10.0 & 82.0 \\
\hline & 6.25 & 6.4 & 0.4 & 6.8 & 101.8 & 6.1 & 0.8 & 13.4 & 97.0 \\
\hline & 12.5 & 12.8 & 0.6 & 4.3 & 102.3 & 11.9 & 1.2 & 10.2 & 95.5 \\
\hline & 18.75 & 19.3 & 0.2 & 0.8 & 103.1 & 18.1 & 1.6 & 8.6 & 96.4 \\
\hline & 25 & 24.1 & 1.3 & 5.4 & 96.4 & 23.0 & 1.0 & 4.2 & 92.1 \\
\hline & 31.25 & 31.2 & 0.2 & 0.5 & 99.9 & 29.6 & 1.9 & 6.3 & 94.8 \\
\hline & 37.5 & 37.7 & 0.5 & 1.4 & 100.7 & 36.1 & 2.2 & 6.1 & 96.3 \\
\hline
\end{tabular}




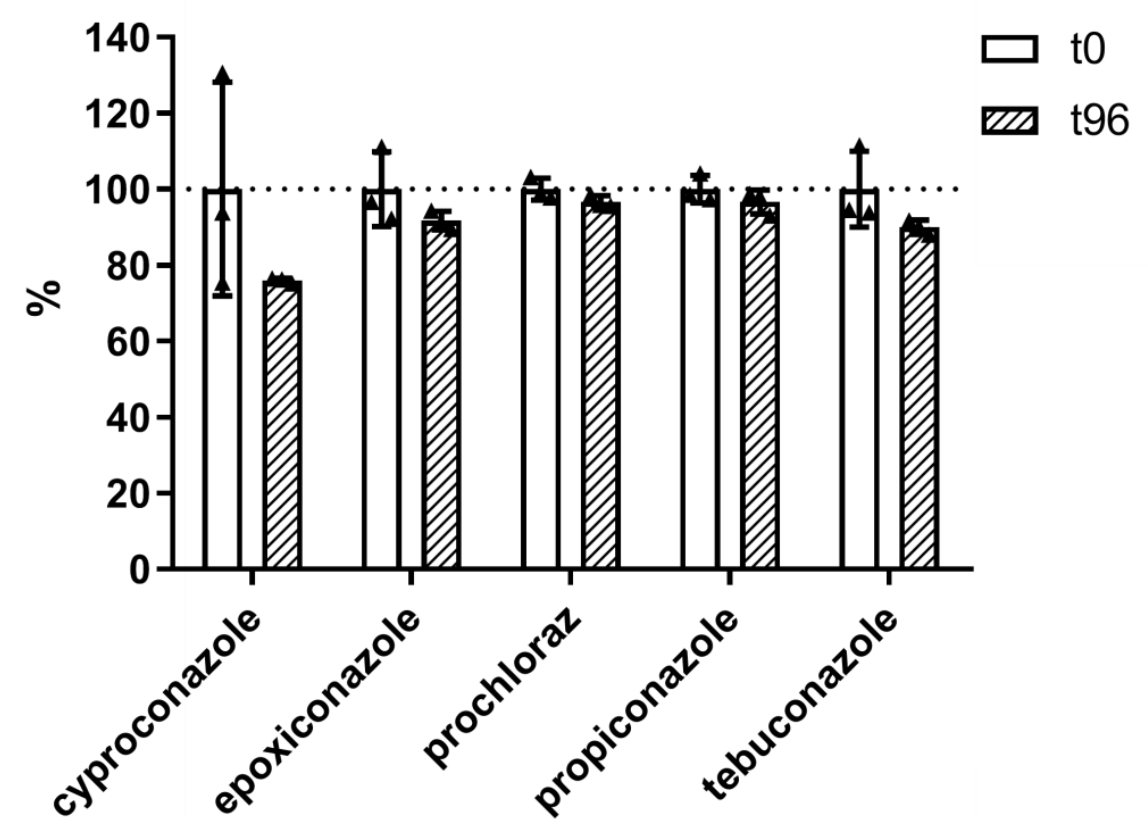

Figure S2: Analytical quantification of azole fungicides: stability after $96 \mathrm{~h}$ treatment in medium and food bacteria. Bars show mean $\pm S D(n=3)$, triangles depict measurements from single experiments.

In the absence of worms, azole fungicides showed recovery rates $>90 \%$ after $96 \mathrm{~h}$ in the test system, except for cyproconazole (76 \%) (Figure S2), which is, however, not due to lower values after $96 \mathrm{~h}$ but instead caused by one unrealistically high concentration value (target concentration $1000 \mathrm{ng} / \mathrm{mL}$, measured single value: $1246.37 \mathrm{ng} / \mathrm{mL}$ ) at $\mathrm{t}=0 \mathrm{~h}$. As we did not detect any decrease of cyproconazole in medium after $96 \mathrm{~h}$ in the presence of worms (Figure S3), we concluded that for all five azole fungicides a high amount of substance degradation and/or biotransformation by food bacteria can be excluded. 


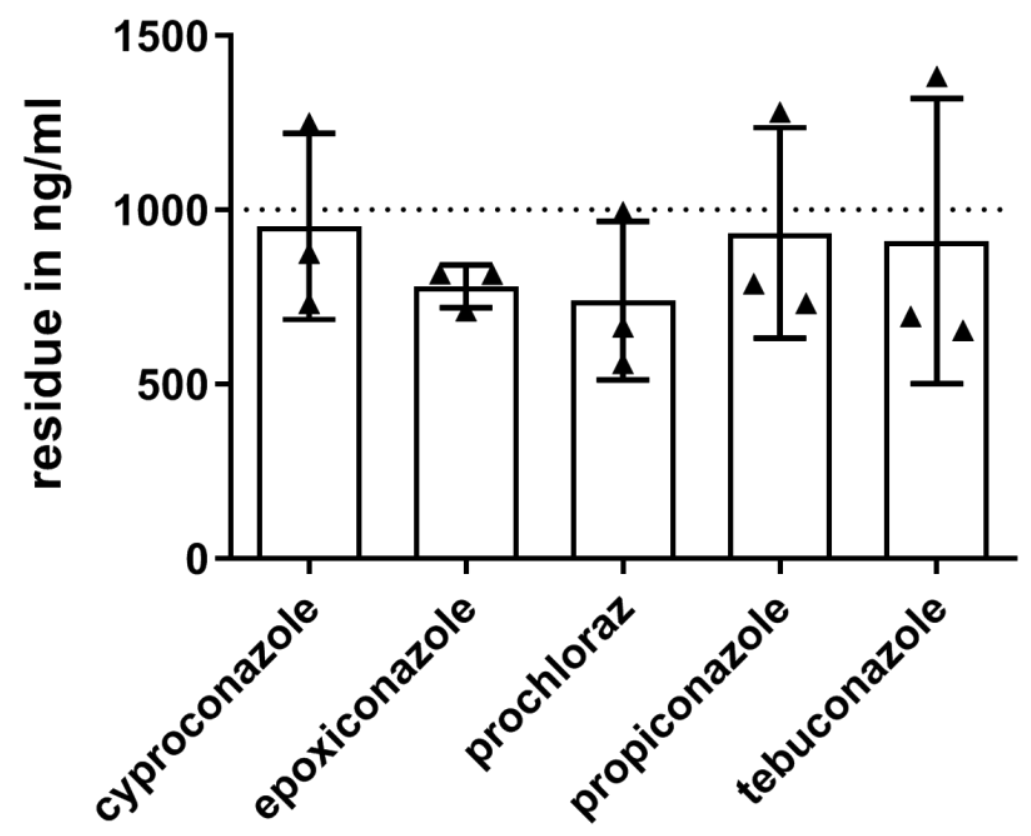

Figure S3: Diagram shows analytical quantified azole fungicide residues in the medium. 400 synchronized L1 were treated with $1 \mu \mathrm{g} / \mathrm{ml}$ azole fungicides for $96 \mathrm{~h}$ in liquid medium. Bars show mean $\pm \mathrm{SD}(\mathrm{n}=3)$ triangles depict measurements from single experiments.

\section{Internal Substance Concentration (Worm)}

In our study, we compared the substance concentrations in worms treated with a specific concentration of azole fungicides to derive: firstly, a measure for the different toxicokinetic properties of the different azoles, i.e. to compare how much substance was actually present in worm tissue (Figure 3, main text), and secondly to test if the substances - when given in binary combinations - interact on a toxicokinetic level (Figure S5).

In preliminary experiments we measured worm tissue concentrations (for detailed method description see S4f, "Quantification and Quality Control") for different medium concentration of each azole (Figure S4). As final test concentration we used $1 \mu \mathrm{g} / \mathrm{mL}$ for each azole because firstly, for all azoles, there were only negligible effects on the growth of the worms and the production of offspring when exposed to this concentration, excluding overlaying secondary effects of toxicity. And secondly, for this concentration linear relationships 
between concentrations in the medium and in the worm for all five substances can be assumed according to preliminary experiments (Figure S4).

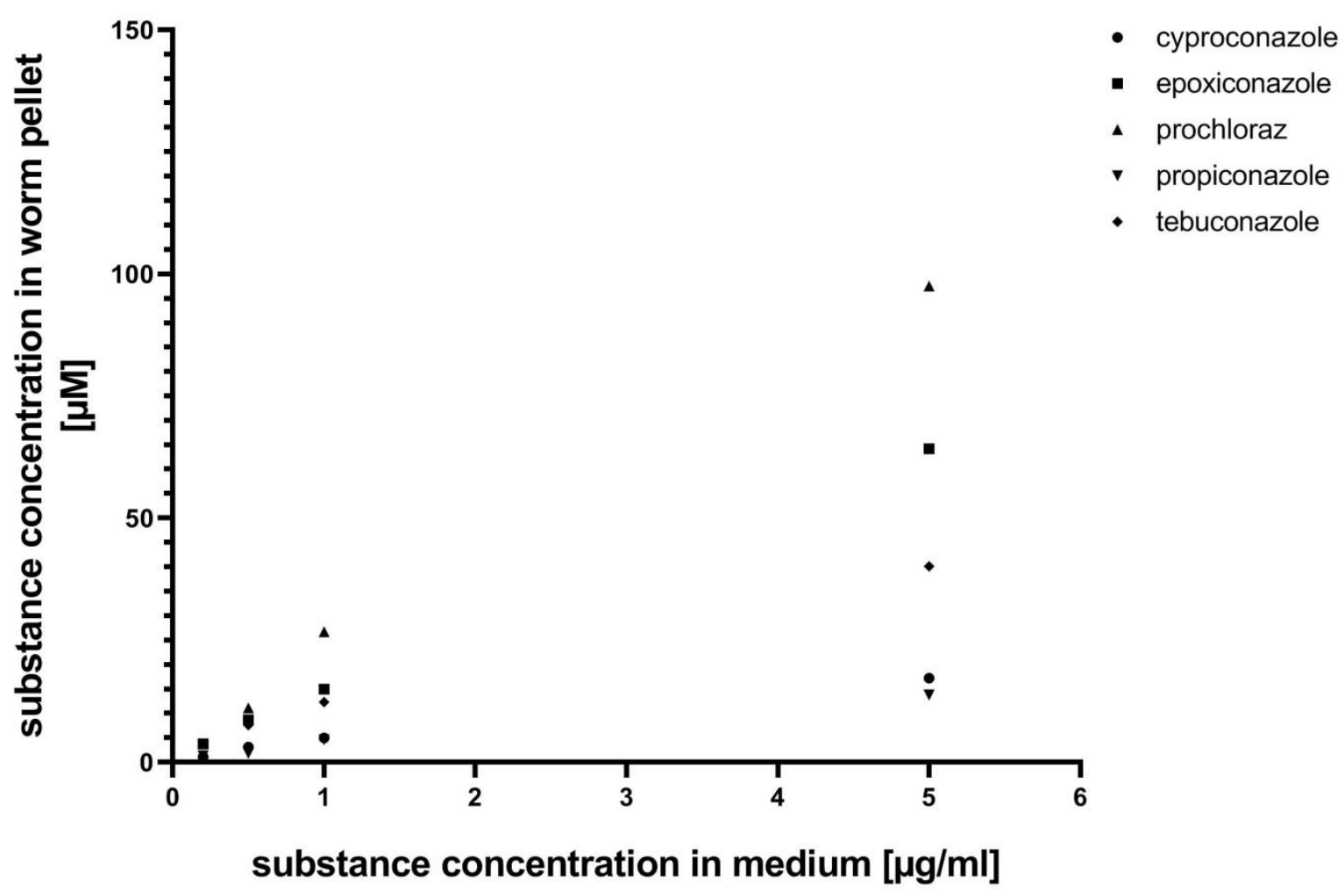

Figure S4: Diagram show azole fungicide concentrations in worm pellets $(n=1) .400$ synchronized L1 were treated with azole fungicides in four concentrations $(0.2,0.5,1$ and $5 \mu \mathrm{g} / \mathrm{mL})$ for $96 \mathrm{~h}$ in liquid medium, azole amount was determined analytically in washed worm pellets. 
cyproconazole

A

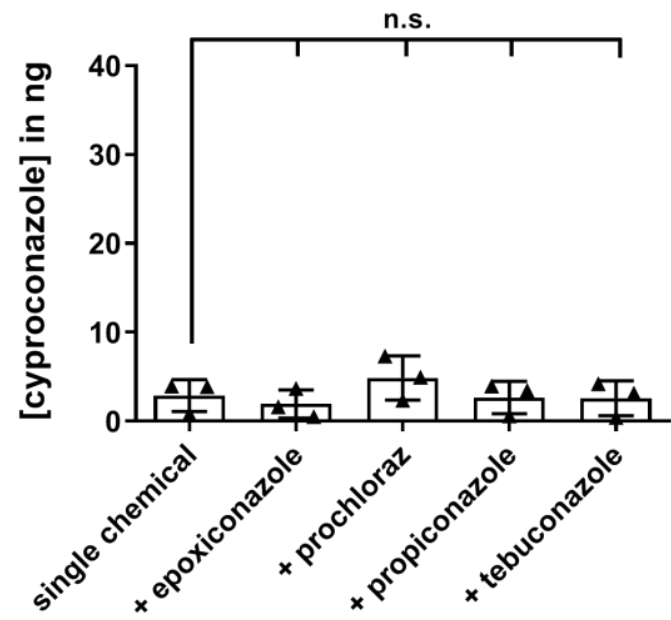

prochloraz

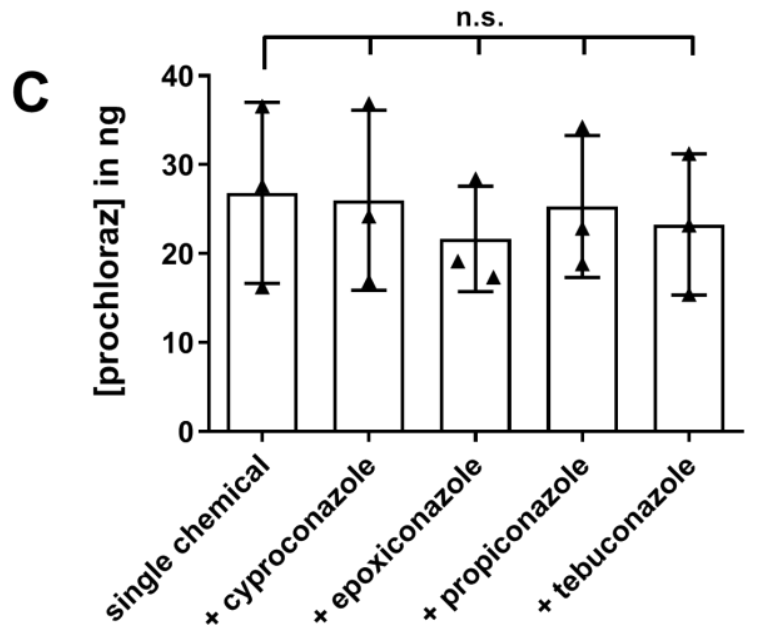

tebuconazole

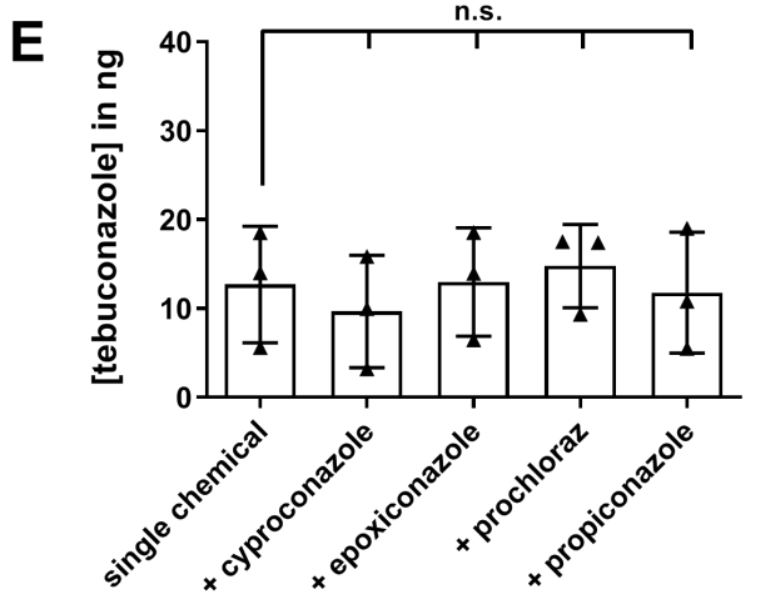

epoxiconazole
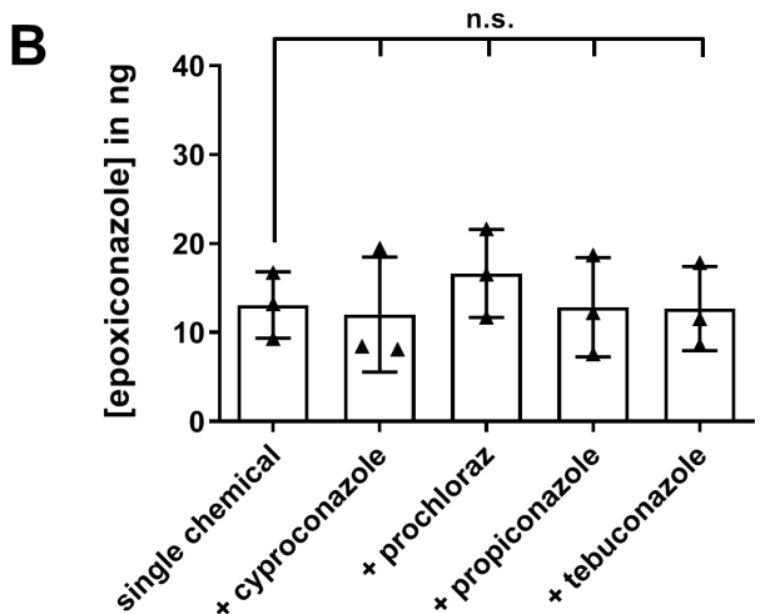

propiconazole

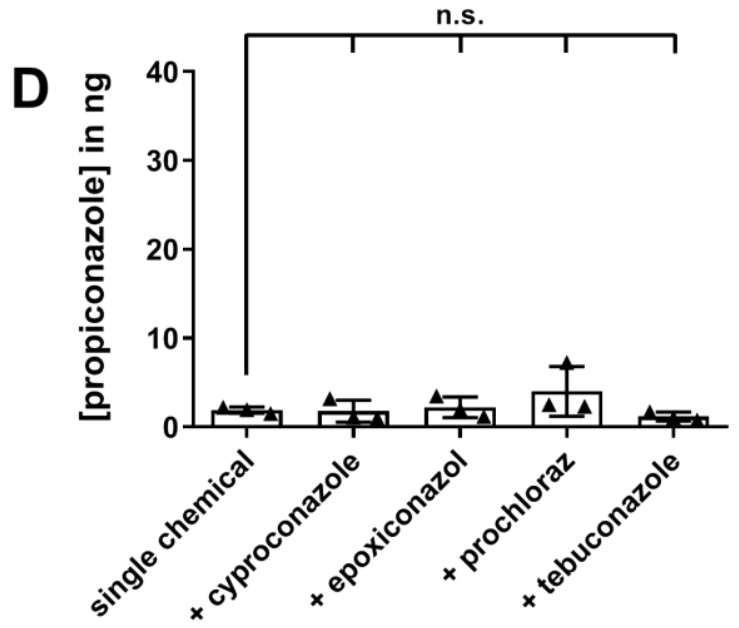

Figure S5: Diagrams show analytical quantified residues of (A) epoxiconazole, (B) prochloraz, (C) cyproconazole, (D) propiconazole and (E) tebuconazole in the worm pellets, related to single chemicals. 400 synchronized L1 were treated with $1 \mu \mathrm{g} / \mathrm{mL}$ azole fungicides alone and in binary combinations for 
$96 \mathrm{~h}$ in liquid medium. Bars show mean \pm SD $(n=3)$, triangles depict measurements from single experiments. Statistical analysis against single chemicals via one-way-ANOVA with Dunnett correction $(p \leq 0.05)$

\section{Prediction of Mixture Effects using CA Model}

Table S9: $\mathrm{IC}_{50}$ for growth and reproduction in $C$. elegans, induced by binary azole fungicide mixtures

\begin{tabular}{ccc}
\hline mixture & growth & offspring \\
\cline { 2 - 3 } & $\mathrm{IC}_{50}$ in $\mu \mathrm{M}[95 \% \mathrm{Cl}]$ & $\mathrm{IC}_{50}$ in $\mu \mathrm{M}[95 \% \mathrm{Cl}]$ \\
epoxiconazole + prochloraz & $14[12.6-15.7]$ & $4[3.2-5.0]$ \\
epoxiconazole + cyproconazole & $200[183-215]$ & $61[51-73]$ \\
epoxiconazole + propiconazole & $214[202-228]$ & $142[129-155]$ \\
epoxiconazole + tebuconazole & $80[74-87]$ & $7[5.5-8.4]$ \\
prochloraz + tebuconazole & $79[74-84]$ & $17[14-22]$ \\
cyproconazole + tebuconazole & $230[215-245]$ & $39[31-48]$ \\
propiconazole + tebuconazole & $189[177-201]$ & $124[107-143]$ \\
\hline
\end{tabular}




\section{epoxiconazole + cyproconazole}
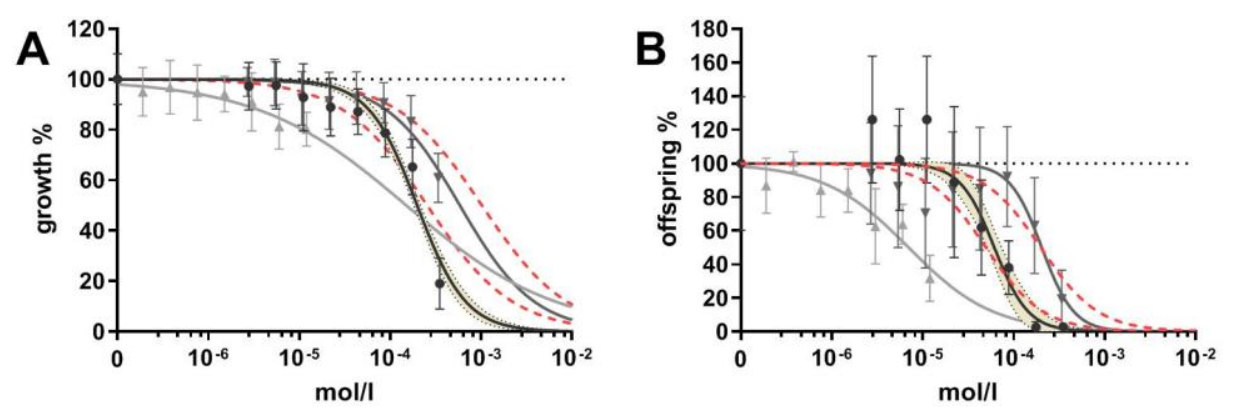

$\rightarrow$ mixture

- epoxiconazole

- cyproconazole

-.. 0.5 CA - 2 CA

\section{epoxiconazole + propiconazole}
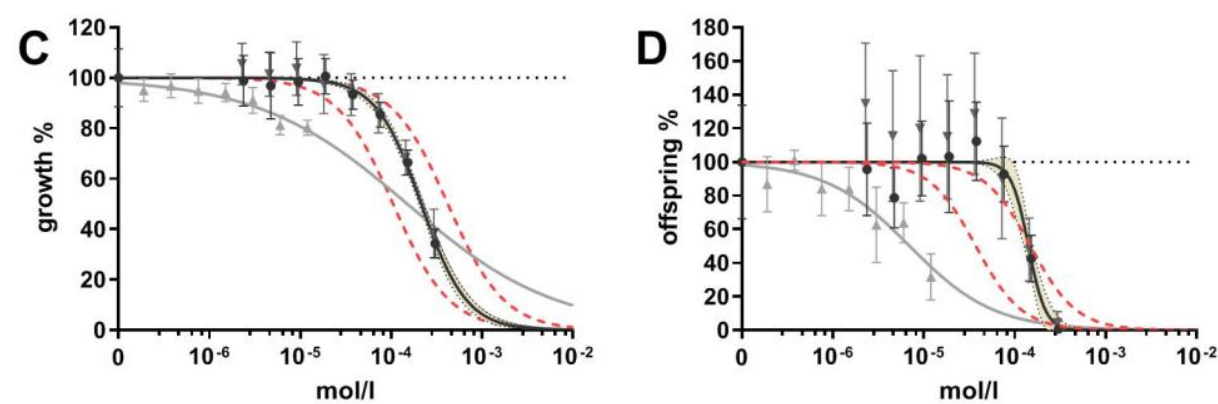

\section{$\rightarrow$ mixture}

- epoxiconazole

* propiconazole

-. 0.5 CA - 2 CA

\section{epoxiconazole + tebuconazole}
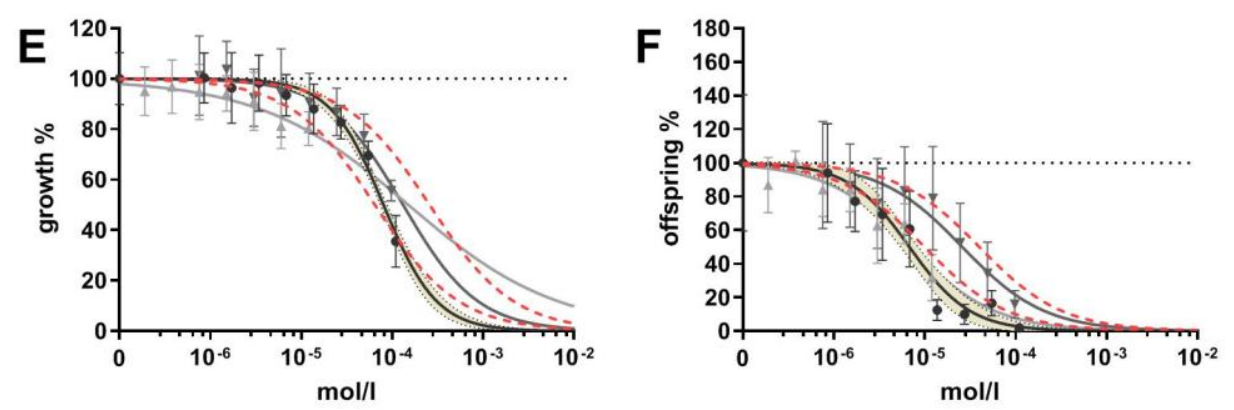

$\rightarrow$ mixture

- epoxiconazole

* tebuconazole

-. 0.5 CA - 2 CA

\section{epoxiconazole + prochloraz}
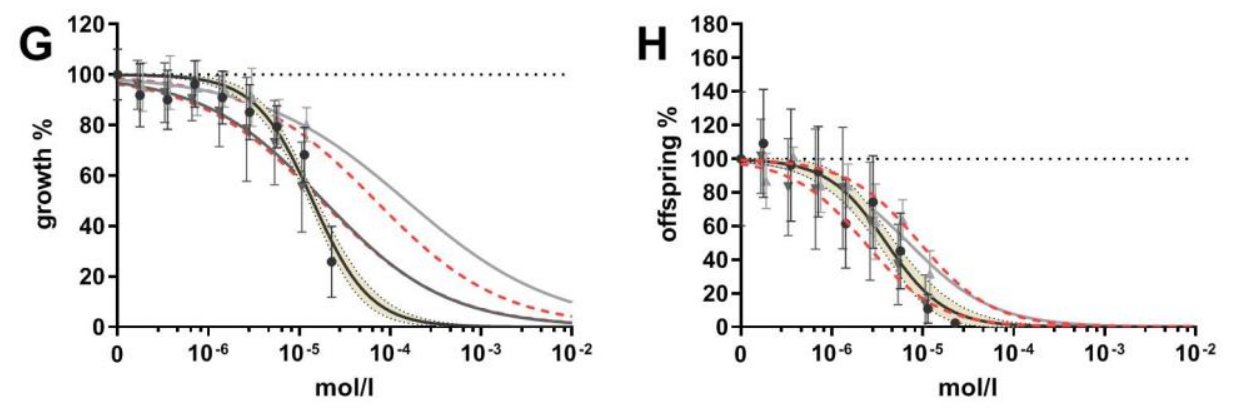

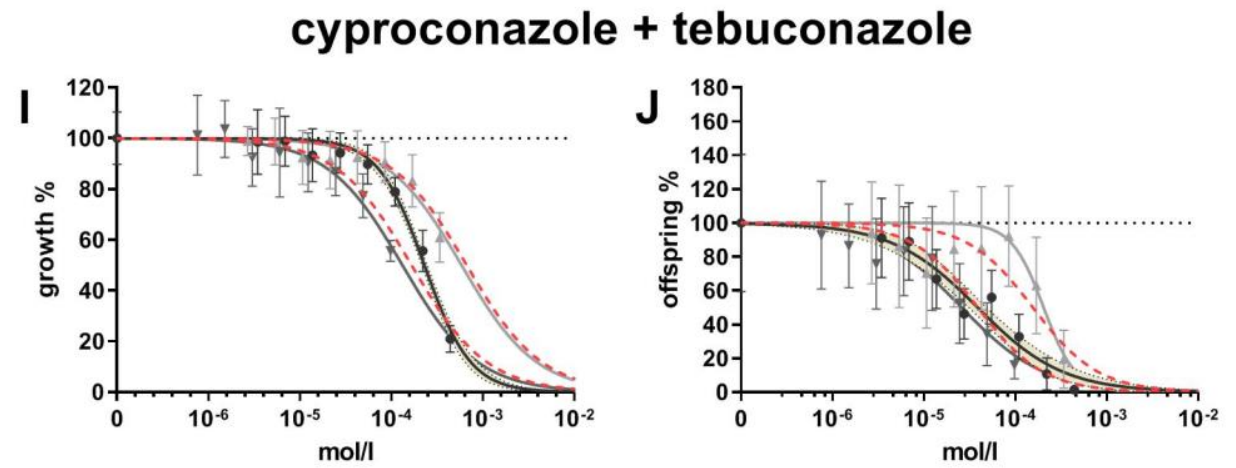
$\rightarrow$ mixture
- cyproconazole
- tebuconazole
-.. 0.5 CA - 2 CA

\section{propiconazole + tebuconazole}
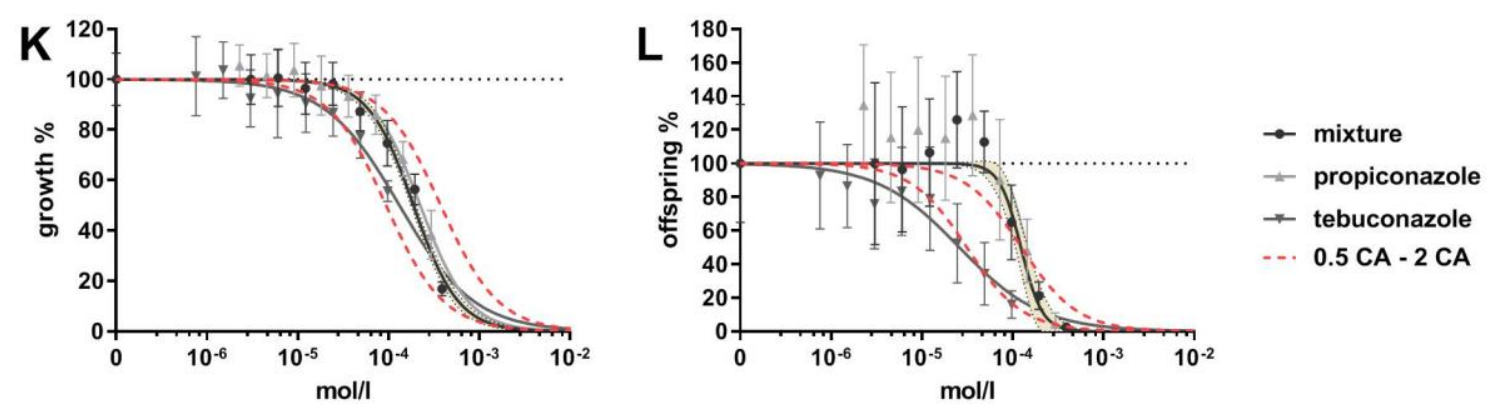

\section{prochloraz + tebuconazole}
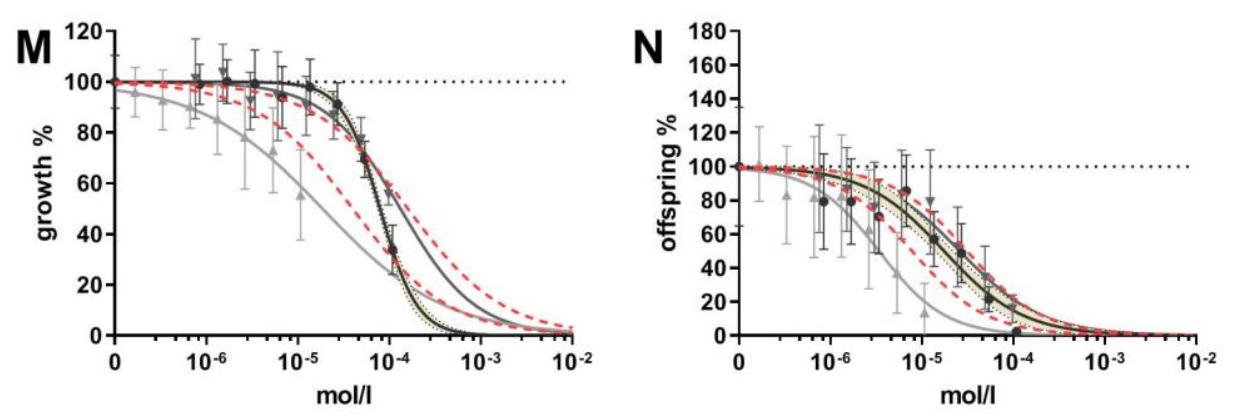
$\rightarrow$ mixture
$\rightarrow$ prochloraz
- tebuconazole
-.. 0.5 CA - 2 CA

Figure S6: Graphs show concentration-response relationships of growth (A, C, E, G, I, K, M) and offspring count (B, D, F, H, J, L, N), relative to solvent controls (DMSO). Synchronized L1 were treated individually with azole fungicides (gray curves: single chemicals; black curves: binary mixtures) for $96 \mathrm{~h}$ in multi-well plates. Mixture effects were predicted using concentration addition (CA) model. Prediction curves were multiplied by factor 0.5 and 2 (red dotted curves) and compared to $95 \%$ confidence intervals of mixture curves (light brown area between black dotted lines). Curves were modelled using GraphPad Prism (log(Inhibitor) vs. normalized response - variable slope). Points show mean \pm SD ( $\geq 13$ per point). 
Table S10: Comparison of azole fungicide binary mixture curves observed experimentally and predicted by $\mathrm{CA}^{\mathrm{a}}$

\begin{tabular}{ccccc}
\hline & \multicolumn{2}{c}{ Growth } & \multicolumn{2}{c}{ Offspring } \\
\cline { 2 - 5 } mixture & accordance & MDR IC $_{50}$ & accordance & MDR IC $_{50}$ \\
\hline epoxiconazole + prochloraz & + & 2.47 & ++ & 1.17 \\
epoxiconazole + cyproconazole & + & 2.58 & ++ & 1.62 \\
epoxiconazole + propiconazole & ++ & 0.98 & + & 0.53 \\
epoxiconazole + tebuconazole & + & 1.61 & + & 2.94 \\
prochloraz + cyproconazole & + & 2.61 & ++ & 1.48 \\
prochloraz + propiconazole & ++ & 1.03 & ++ & 0.85 \\
prochloraz + tebuconazole & + & 1.01 & ++ & 0.89 \\
cyproconazole + propiconazole & ++ & 1.05 & ++ & 0.75 \\
cyproconazole + tebuconazole & ++ & 1.45 & ++ & 2.01 \\
propiconazole + tebuconazole & ++ & 1.00 & + & 0.51 \\
\hline
\end{tabular}

${ }^{a} 95 \%$ confidence intervals of experimentally determined azole fungicide binary mixture curves were compared to tolerance range (factor $0.5-2$ ) of calculated concentration addition (CA) predictions (++ good accordance; + partial accordance). Model deviation ratios (MDR) were calculated at half-maximal inhibitory concentration $\left(\mathrm{IC}_{50}\right)$.

Table S11: Normalization of the measured effect concentrations to the ratio between the internal and the respective concentration surrounding liquid medium

\begin{tabular}{cccccc}
\hline & \begin{tabular}{c} 
relative \\
concentration \\
\cline { 3 - 6 }
\end{tabular} & \multicolumn{2}{c}{ Growth } & \multicolumn{2}{c}{ Offspring } \\
\cline { 3 - 6 } & $1 \%]^{\mathrm{a}}$ & $\mathrm{IC}_{50}[\mu \mathrm{M}]^{\mathrm{b}}$ & adj. IC $\mathbf{C}_{50}[\mu \mathrm{M}]^{\mathrm{c}}$ & $\mathrm{IC}_{50}[\mu \mathrm{M}]^{\mathrm{b}}$ & adj. IC $\mathbf{C}_{50}[\mu \mathrm{M}]^{\mathrm{c}}$ \\
\hline cyproconazole & 144 & 586 & 846 & 204 & 295 \\
epoxiconazole & 655 & 151 & 988 & 7 & 46 \\
prochloraz & 1341 & 19 & 255 & 3 & 40 \\
propiconazole & 96 & 223 & 214 & 141 & 135 \\
tebuconazole & 636 & 132 & 839 & 26 & 165 \\
\hline
\end{tabular}

${ }^{a}$ Rate of internal concentration to concentration in liquid medium assuming a volume of $5 \mathrm{~nL}$ per adult worm

${ }^{\mathrm{b}} \mathrm{IC}_{50}$ for growth and offspring value for nominal concentration in liquid medium

${ }^{c}$ adjusted $\mathrm{IC}_{50}$ for growth and offspring, i.e. value for estimated internal concentration 
Table S12: rat data from a previously published study $\mathrm{y}^{2,3}$

\begin{tabular}{ccccccc}
\hline & $\begin{array}{c}\text { External } \\
\text { dose } \\
{[\mathbf{p p m}]}\end{array}$ & $\begin{array}{c}\text { Liver } \\
\text { residue } \\
{[\mathrm{mg} / \mathbf{k g}]}\end{array}$ & $\begin{array}{c}\text { bw at } \\
\text { termination } \\
{[\mathbf{g}]}\end{array}$ & $\begin{array}{c}\text { Mean daily } \\
\text { substance } \\
\text { intake } \\
{[\mathbf{m g} / \mathbf{k g} \text { bw] }}\end{array}$ & $\begin{array}{c}\text { Mean daily } \\
\text { substance } \\
\text { intake } \\
{[\mathrm{mg}]}\end{array}$ & $\begin{array}{c}\text { Relative } \\
\text { liver } \\
\text { weight } \\
\text { change } \\
{[\%]}\end{array}$ \\
\hline cyproconazole & 1000 & 15.6 & 411.6 & 74.00 & 30.5 & 45 \\
epoxiconazole & 900 & 8.22 & 423.2 & 61.79 & 26.1 & 20 \\
prochloraz & 1000 & 3.46 & 425.8 & 68.22 & 29.0 & 15 \\
propiconazole & 2400 & 0.45 & 447.2 & 181.19 & 81.0 & 20 \\
tebuconazole & 1000 & 1.80 & 458.2 & 71.24 & 32.6 & 7 \\
\hline
\end{tabular}

\section{References}

1. So, S.; Miyahara, K.; Ohshima, Y., Control of body size in C. elegans dependent on food and insulin/IGF-1 signal. Genes to cells : devoted to molecular \& cellular mechanisms 2011, 16, (6), 63951; DOI 10.1111/j.1365-2443.2011.01514.x.

2. Schmidt, F.; Marx-Stoelting, P.; Haider, W.; Heise, T.; Kneuer, C.; Ladwig, M.; Banneke, S.; Rieke, S.; Niemann, L., Combination effects of azole fungicides in male rats in a broad dose range. Toxicology 2016, 355-356, 54-63; DOI 10.1016/j.tox.2016.05.018.

3. Heise, T.; Schmidt, F.; Knebel, C.; Rieke, S.; Haider, W.; Pfeil, R.; Kneuer, C.; Niemann, L.; Marx-Stoelting, P., Hepatotoxic effects of (tri)azole fungicides in a broad dose range. Archives of toxicology 2015, 89, (11), 2105-17; DOI 10.1007/s00204-014-1336-1. 